\title{
Cancer Cell-specific Transfection of hCas9 Gene Using Ad5F35 Vector
}

\author{
WATARU MATSUNAGA ${ }^{1}$, KATSUYUKI HAMADA ${ }^{2,3}$, MASATOSHI TAGAWA $^{4}$, \\ TAKAO MORINAGA ${ }^{5}$ and AKINOBU GOTOH ${ }^{6}$ \\ ${ }^{1}$ Joint-use Research Facilities, Hyogo College of Medicine, Nishinomiya, Japan; \\ ${ }^{2}$ Department of Clinical Oncology, School of Medicine, Toho University, Tokyo, Japan; \\ ${ }^{3}$ Department of Obstetrics and Gynecology, School of Medicine, Ehime University, Ehime, Japan; \\ ${ }^{4}$ Department of Biochemistry and Genetics, Graduate School of Medicine, Chiba University, Chiba, Japan; \\ ${ }^{5}$ Division of Pathology and Cell Therapy, Chiba Cancer Center Research Institute, Chiba, Japan; \\ ${ }^{6}$ Department of Education for Medical Research Base, Hyogo College of Medicine, Nishinomiya, Japan
}

\begin{abstract}
Background: The clustered regularly interspaced short palindromic repeats-associated protein 9 (CRISPR-Cas9) is thought to have promising clinical potential. However, the off-target effects of Cas9 are a major concern for its application. Therefore, we hypothesized that the adverse effects of off-target gene editing might be minimized if the human codon-optimized Streptococcus pyogenes Cas9 (hCas9) could be specifically expressed in cancer cells. Materials and Methods: We constructed a chimeric adenoviral vector, Ad5F35-MKp-hCas9, and infected human bladder cancer cell lines with this vector. The confirmation of hCas 9 gene expression was performed in 3-4 days after from infection. Results: hCas 9 gene expression was observed in Ad5F35-MKphCas 9 infected bladder cancer cells but not in non-malignant cells. Conclusion: Our study showed that the Ad5F35-MKp$h$ Cas 9 vector is capable of expressing the hCas 9 gene with high specificity in bladder cancer cells. These findings may help in minimizing the risk of off-target effects of gene editing.
\end{abstract}

Non-muscle invasive bladder cancer (NMIBC) accounts for approximately $70 \%$ of all bladder cancer. Although patients with bladder cancer have a relatively low mortality rate (1), most NMIBC cases develop recurrent tumors and progress to a higher stage or grade (2), thus making NMIBC treatment crucial. At present, Bacillus Calmette-Guérin therapy is

This article is freely accessible online.

Correspondence to: Akinobu Gotoh, Department of Education for Medical Research Base, Hyogo College of Medicine, 1-1 Mukogawa-cho, Nishinomiya, 663-8501, Japan. Tel: +81 798456807, Fax: +81 798456806, e-mail: gotoh@hyo-med.ac.jp

Key Words: Adenovirus vector, Cas9, midkine, bladder cancer. considered to be the most effective treatment for refractory NMIBC; however, its severe adverse effects significantly reduce the patient's quality of life and cause half of all patients to abandon treatment (3). Therefore, development of new treatments that do not reduce patients' quality of life is very important in the therapy of NMIBC.

In previous studies, we focused on virotherapy for the development of novel NMIBC treatment, and examined the oncolytic effect of the adenoviral vector (4) and the survival inhibition effect of lentiviral vector-mediated gene transfection (5) on various bladder cancer cell lines. We also developed the Ad5F35 vector, which is a chimeric adenoviral vector of serotype $5(\operatorname{Ad} 5)$ and serotype $35(\operatorname{Ad} 35)$ adenovirus $(4,6,7)$. The Ad5F35 vector is a conditionally replicating adenovirus which infects via CD46, which is a surface antigen that is highly expressed in bladder cancer cells (4). Furthermore, the E1 gene is placed under the promoter of midkine, which is a highly expressed growth factor in cancer cells (8). Therefore, it is difficult for Ad5F35 to infect cells other than cancer cells and grow in non-malignant cells.

However, adenoviruses lead to the formation of antibodies immediately after infection or administration. Neutralizing antibodies to Ad5 are quite common in humans $(9,10)$, and pre-existing immunity to adenovirus is thought to result in ineffective delivery of administered adenovirus $(11,12)$. While a previous study claimed that pre-immunity to viruses does not diminish the viral oncolytic effect (13), recent studies suggest that pre-immunity is a major obstacle to the adenovirus vector $(14,15)$. Taken together, the possibility that the therapeutic effect is diminished at an early stage by the neutralizing antibody is a serious concern when considering the clinical application of Ad5F35 in cancer therapy. Therefore, to obtain a higher therapeutic effect in a short period using Ad5F35, we considered that a new function is required. 
The clustered regularly interspaced short palindromic repeats-associated protein 9 (CRISPR-Cas9) system is based on the immune system in Streptococcus pyogenes that protects bacterial cells from foreign DNA $(16,17)$, and this system has been utilized as a versatile gene-editing tool (18). CRISPR-Cas9 can cleave any complementary site to the guide RNA in the DNA double strands, thus making gene editing dramatically easier. At present, CRISPR-Cas9 technology is considered to be a promising new treatment for many human diseases through modification of specific genes or repair of mutations. In addition, in the field of cancer therapy, CRISPR-Cas9 has shown excellent clinical potential (19, 20). In 2016, the Food and Drug Administration approved the first clinical trial of cancer treatment using CRISPR-Cas9, in which T-cells were extracted from a patient, altered by CRISPR-Cas9, and finally returned to the patient (21).

However, the CRISPR-Cas9 system may cause unintended DNA modifications. This off-target effect occurs relatively frequently, and is a major concern for the clinical applications of CRISPR-Cas9 (22-24). Various studies have been performed to control the off-target effects of CRISPRCas9 (25-27); however, total control of off-target effects is thought to be very difficult. We hypothesized that if it were possible to specifically express Cas9 gene in cancer cells, the adverse effects or risks of off-target gene editing could be minimized.

We constructed a chimeric Ad5F35 vector containing the Cas 9 gene regulated by the midkine promoter to restrict infection and gene expression in cancer cells, and examined cancer cell-specific Cas9 gene transfection using this vector.

\section{Materials and Methods}

Cells and cell culture. In this study, three human bladder cancer cell lines were used, 5637, UMUC3, and 253J. These cell lines were purchased from the American Type Culture Collection (Manassas, VA, USA). The human prostatic cell line PNT1A (Public Health England, Porton Down, UK) was used as a non-malignant cell line. In addition, HEK293 cells (American Type Culture Collection) were used as packaging cells and as a positive control for vector infection.

The cell lines 5637 and PNT1A were grown in Roswell Park Memorial Institute 1640 medium (RPMI1640; Life Technologies Co., NY, USA), and UMUC3, 253J, and HEK293 cells were grown in Dulbecco's modified Eagle's medium (Life Technologies). Both media were supplemented with $10 \%$ heat-inactivated fetal bovine serum (FBS; BioWest, Nuaillé, France) and $100 \mathrm{U} / \mathrm{ml}$ penicillinstreptomycin (Nacalai Tesque Inc., Kyoto, Japan). The cultures were maintained in humidified $5 \% \mathrm{CO}_{2}$ and $95 \%$ air at $37^{\circ} \mathrm{C}$.

Construction of vector. A chimeric adenoviral vector of type 5 and type 35 adenovirus, Ad5F35-MKp-hCas9, was used in this study. The components of the adenovirus plasmid used for this vector are shown in Figure 1A. The 609-bp 5'- upstream regulatory region of the midkine gene (28) was used as a promoter (Mkp: Figure 1B).
The human codon-optimized Streptococcus pyogenes Cas9 gene (hCas9; Figure 1C) was placed downstream of the Mkp, and the enhanced green fluorescent protein (EGFP) gene was inserted next to the hCas9 gene as an expression marker. In addition, two adenoviral vectors were used as controls in this experiment; one was Ad5F35-CMVp-hCas9, which encodes hCas9 and EGFP genes under the cytomegalovirus promoter (CMVp), and the other was Ad5-CMVp-EGFP, which encoded EGFP under CMVp. Plasmid construction was ordered from VectorBuilder (Chicago, IL, USA).

3-(4,5-Dimethylthiazol-2-yl)-2,5-diphenyltetrazolium bromide (MTT) assay. Infection with Ad5F35-MKp-hCas9 was performed at 0, 10, 100 , and 1,000 infective units/cell [multiplicity of infection (MOI)] at an initial density of $30,000 \mathrm{cells} / \mathrm{ml}$, and 0 MOI was used as a negative control. After infection, cells were seeded into 96-well plates $(3,000$ cells/well, medium containing 5\% FBS $)$ and incubated in $5 \% \mathrm{CO}_{2}$ and $95 \%$ air at $37^{\circ} \mathrm{C}$ for 4 days. The cell viability rate of each cell line was measured using the MTT assay. Four days after infection, $250 \mu \mathrm{g} / \mathrm{ml}$ of MTT (Dojin Kagaku, Kumamoto, Japan) was dissolved in serum-free Dulbecco's modified Eagle's medium or RPMI1640, which was used to replace the 96-well plate incubation medium $(100 \mu \mathrm{L} /$ well $)$, and further incubated at $37^{\circ} \mathrm{C}$ in $5 \% \mathrm{CO}_{2}$ and $95 \%$ air for $4-6 \mathrm{~h}$. The stop solution (20\% sodium dodecyl sulfate and $50 \% \mathrm{~N}, \mathrm{~N}$-dimethylformamide, $\mathrm{pH} 4.0$ ) was added to each well $(100 \mu \mathrm{l} /$ well $)$ and incubated overnight at room temperature with mechanical shaking. The absorbance was then measured using SpectraMax ${ }^{\circledR}$ Plus 384 (Molecular Devices, San Jose, CA, USA), as described previously (29).

To compare the different MOIs of the vectors against each respective cell line, we used a one-way analysis of variance or Kruskal-Wallis rank-sum test followed by Tukey's post-hoc test. Differences were considered significant at $p<0.05$.

Western blotting. Infection with Ad5F35-MKp-hCas9 was performed at 0 (negative control), 10 , and 100 MOI at an initial density of 300,000 cells $/ \mathrm{ml}$. Infected cells were seeded in a $6 \mathrm{~cm}$ dish and incubated in $5 \% \mathrm{CO}_{2}$ and $95 \%$ air at $37^{\circ} \mathrm{C}$ for 4 days. After infection for $48 \mathrm{~h}$, the medium was replaced with a vector-free medium containing 5\% FBS, and cells were further incubated for $48 \mathrm{~h}$. Cells were then lysed in electrophoresis sample buffer $(500 \mu \mathrm{l} / \mathrm{dish})$ on crushed ice and stored at $-20^{\circ} \mathrm{C}$ until western blotting. Samples (5$20 \mu \mathrm{l} /$ lane) were separated on an $8 \%$ sodium dodecyl sulfatepolyacrylamide gel electrophoresis gel, and electroblotting was performed on a nitrocellulose membrane (pore size, $0.2 \mu \mathrm{m}$; FUJIFILM Wako Pure Chemical, Osaka, Japan) in EzFastBlot (ATTO, Osaka, Japan). The blotted membranes were pretreated with $1 \%$ skim milk in tris-buffered saline containing $0.5 \%$ Tween 20 (TBST) at $4^{\circ} \mathrm{C}$ overnight. Membranes were then incubated with EPR18991 anti-CRISPR-Cas9 rabbit monoclonal antibody (1/1,500 in TBST, Abcam, Cambridge, UK) overnight at $4^{\circ} \mathrm{C}$, followed by a horseradish peroxidase (HRP)-conjugated anti-rabbit $\operatorname{IgG}(1 / 10,000$ in TBST, Cell Signaling Technology, Boston, USA) and HRPconjugated anti-glyceraldehyde 3-phosphate dehydrogenase (1/20,000 in TBST; Medical \& Biological Laboratories, Aichi, Japan) for $3 \mathrm{~h}$ at room temperature. HRP was visualized using Amersham ECL Prime western blotting Detection Reagents (GE Healthcare, Little Chalfont, UK) according to the manufacturer's protocol.

Polymerase chain reaction (PCR). PCR was performed to detect hCas9 DNA in the infected cells. Infection with Ad5F35-MKp- 
A

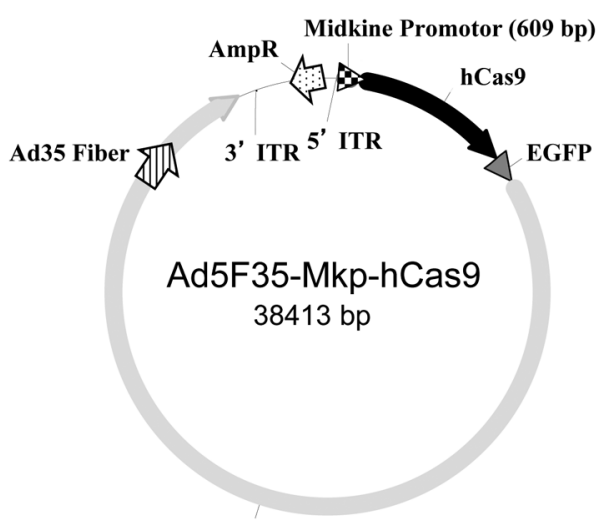

E1/E3 deleted Ad5
B

Sequence of Midkine Promoter

1 GCTTCCCTGCCCACCCGCGGAAACCGCCCCAGGTGGCCGCGCCCCCTCCC 50 51 CAGCAGCCAGCAGGGCGCCAGGGCTGAGCCGGCCGTGGAGGGGAGCGGGT 100 101 CCCGGGGGTTATACAGGCGCCGGGCGTCCGCGGCAGGCAAGAGAAGCTGA 150 151 GGCCTGAGAACGGCCCGGGCCTTGGCGTACGGCAGGGGACGACCTGGGAT 200 201 GGGGGCAGCGGGCGGCGGCGCAGGGAGTGGGCCGGGGCCGGTGTGCGCGG 250 251 GCGGGACGGGGCCGGGGTCGGGAGACCACCGCTCGGAAGATGGGGCCGGG 300 301 AGAGGCCGCCGTCGCAGCGCAGAGGGCACCGGCGGGGAGACGCGAGGACG 350 351 CGGGGCCGGGAACACGGACGCCGGAGTAGAAGCGCGGGGGGGGCGGGCTG 400 401 GAGCGGGGGCGGGGACGCCGGGGTCGGGGGCGGTGCGGGTTTGAGGGGAG 450 451 GGGGCGGGGCGGGTCCTTCCCTGGGGGGGTGGGGAGAGGGGGCGGGGGCC 500 501 CATGTGACCGGCTCAGACCGGTTCTGGAGACAAAAGGGGCCGCGGCGGCC 550 551 GGAGCGGGACGGGCCCGGCGCGGGAGGGAGCGAAGCAGCGCGGGCAGCGA 600 601 GCGAGTGAG 609

$\mathrm{C}$

\section{Sequence of hCas9}

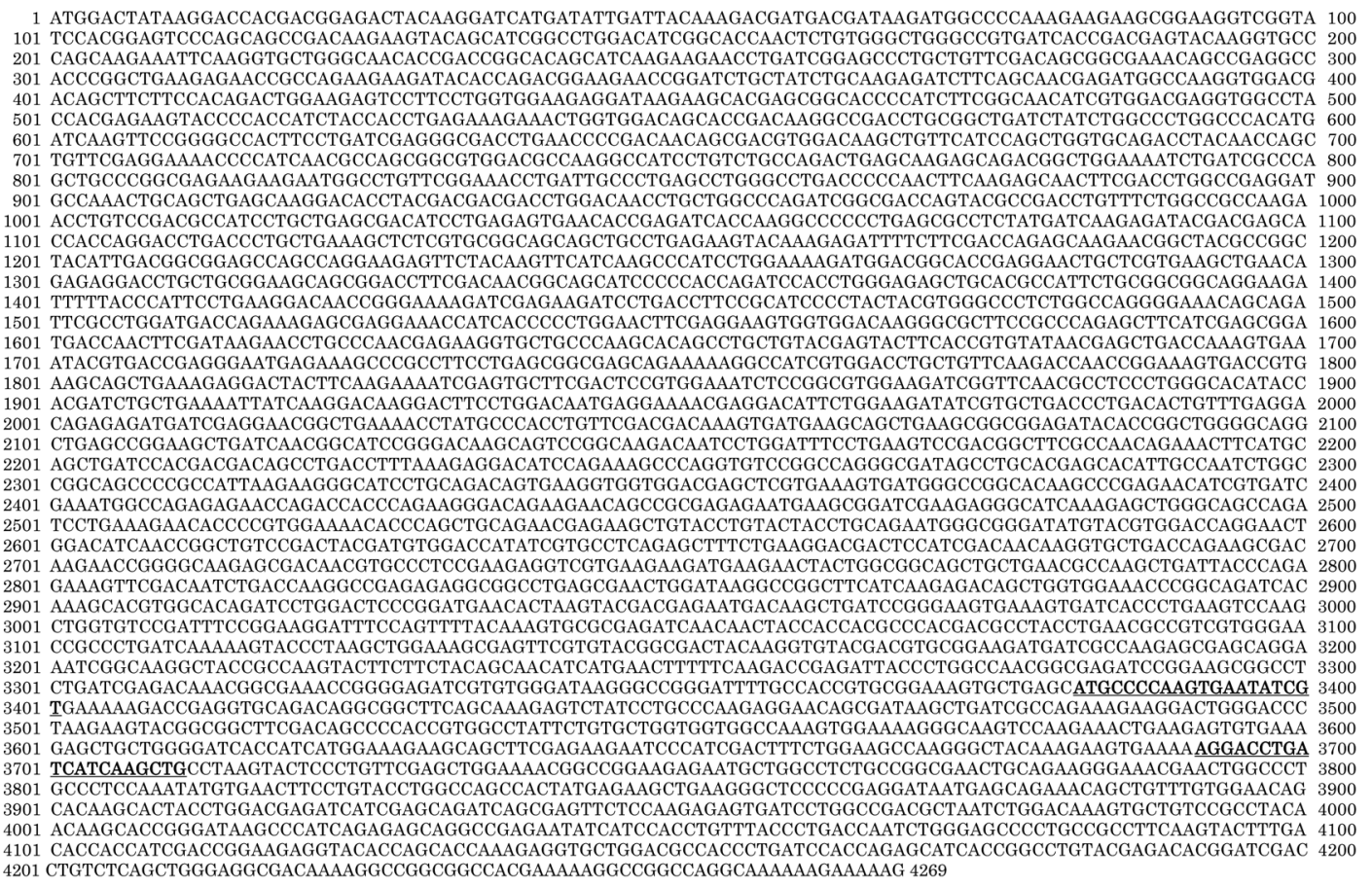

Figure 1. A: Structure of chimeric Ad5F35 vector containing the human codon-optimized Streptococcus pyogenes Cas 9 (hCas 9$)$ gene regulated by the midkine promoter (Ad5F35-Mkp-hCas9). ITR: Inverted terminal repeat sequence; EGFP: enhanced green fluorescent protein gene; Ad35 Fiber: the fiber knob of adenovirus type 35. B: The sequence of midkine promoter used in this study. This is based on the study of Uehara et al. (28). C: The sequence of hCas 9 gene. The bold underlined parts are the sequences of the polymerase chain reaction primer.

hCas 9 was carried out in the same way as described in the western blotting section. Infected cells were incubated in $5 \% \mathrm{CO}_{2}$ and $95 \%$ air at $37^{\circ} \mathrm{C}$ for 3 days. At $48 \mathrm{~h}$ after infection, the medium was replaced with a vector-free medium containing 5\% FBS and cells were further incubated for $24 \mathrm{~h}$. After incubation, cells were collected by centrifugation $\left(190 \times g, 10 \mathrm{~min}\right.$ at $\left.4^{\circ} \mathrm{C}\right)$, followed by removal of the medium and washing of the pellet. Total cell DNA was extracted using a MonoFas gDNA Cultured Cells Extraction Kit VI (ANIMO, Kawaguchi, Japan) according to the manufacturer's protocol. The hCas9-specific primers (forward primer: 5'-ATGCC CCAAGTGAATATCGT-3', reverse primer: 5'-CAGCTTGATGAT CAGGTCCT-3; Figure 1C) were designed using Primer 3, and the 

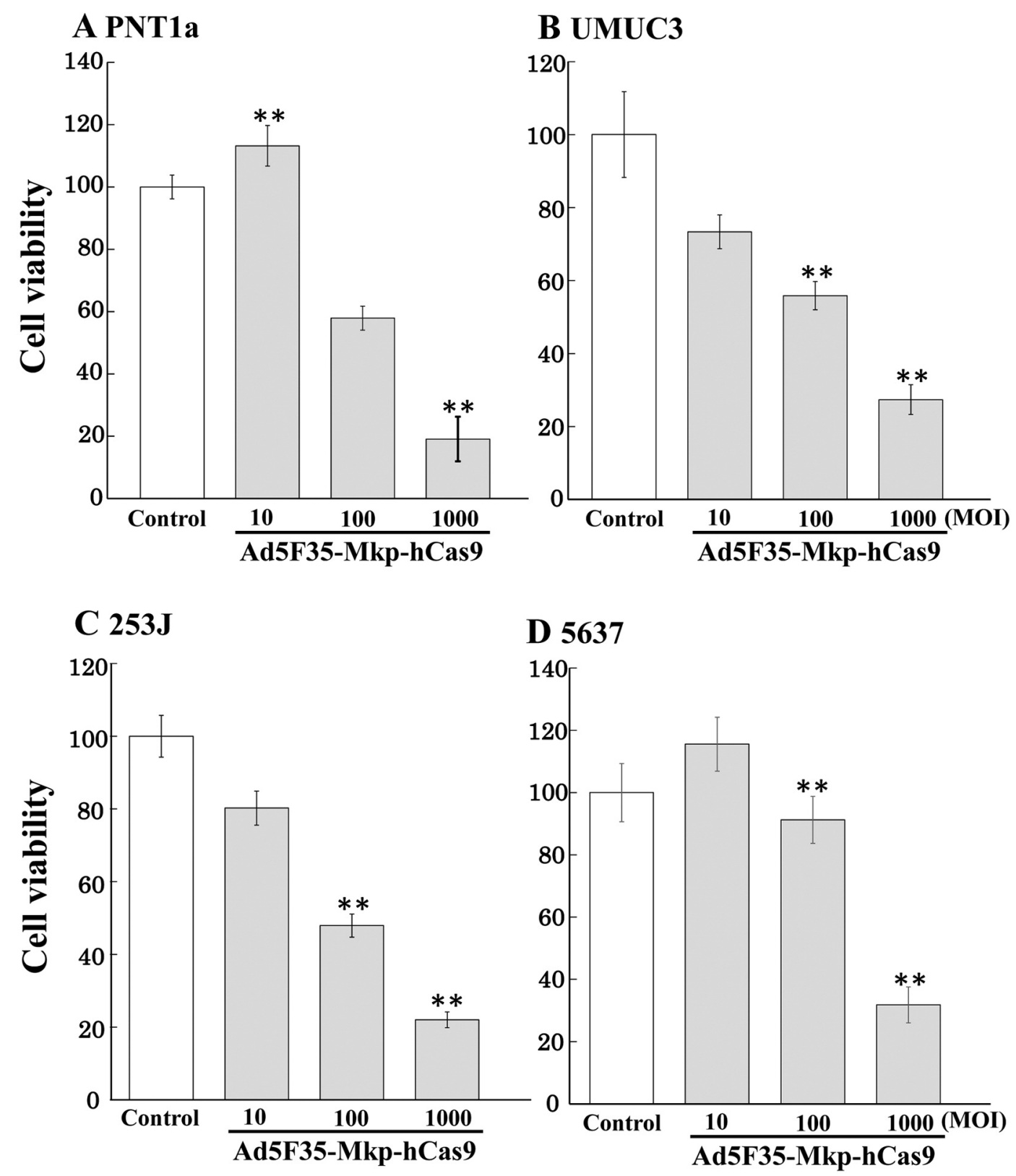

Figure 2. Cytotoxicity of chimeric Ad5F35 vector containing the Cas 9 gene regulated by the midkine promoter (Ad5F35-Mkp-hCas9) towards nonmalignant cells (PNT1A; A) and human bladder cancer cell lines (UMUC3; B, 253J; C and 5637; D). Cell viability was measured using 3-(4,5dimethylthiazol-2-yl)-2,5-diphenyltetrazolium bromide assay 4 days after infection. Data are the mean \pm standard error as a percentage of control cells (uninfected cells). **Significantly different at $p<0.01$. MOI: Multiplicity of infection.

length of the PCR product was 329 bp. The PCR reaction was performed using Tks Gflex ${ }^{\mathrm{TM}}$ DNA Polymerase (Takara Bio, Kusatsu, Japan) according to the following protocol: $94^{\circ} \mathrm{C}$ for 1 min, followed by $15-23$ cycles of $95^{\circ} \mathrm{C}$ for $10 \mathrm{~s}, 55^{\circ} \mathrm{C}$ for $15 \mathrm{~s}$, and $68^{\circ} \mathrm{C}$ for $30 \mathrm{~s}$. The amplified products were electrophoretically separated on a $2 \%$ agarose gel, and the amplified products were visualized with ethidium bromide and a UV transilluminator. At least two experiments were performed to assess reproducibility.

\section{Results}

Effect of Ad5F35-Mkp-hCas9 on cell viability. Figure 2 shows the cell viability rate measured by the MTT assay in experimental cells $96 \mathrm{~h}$ after infection with Ad5F35Mkp-hCas9. In the case of the non-malignant cell line PNT1A, significant survival inhibition was observed at 


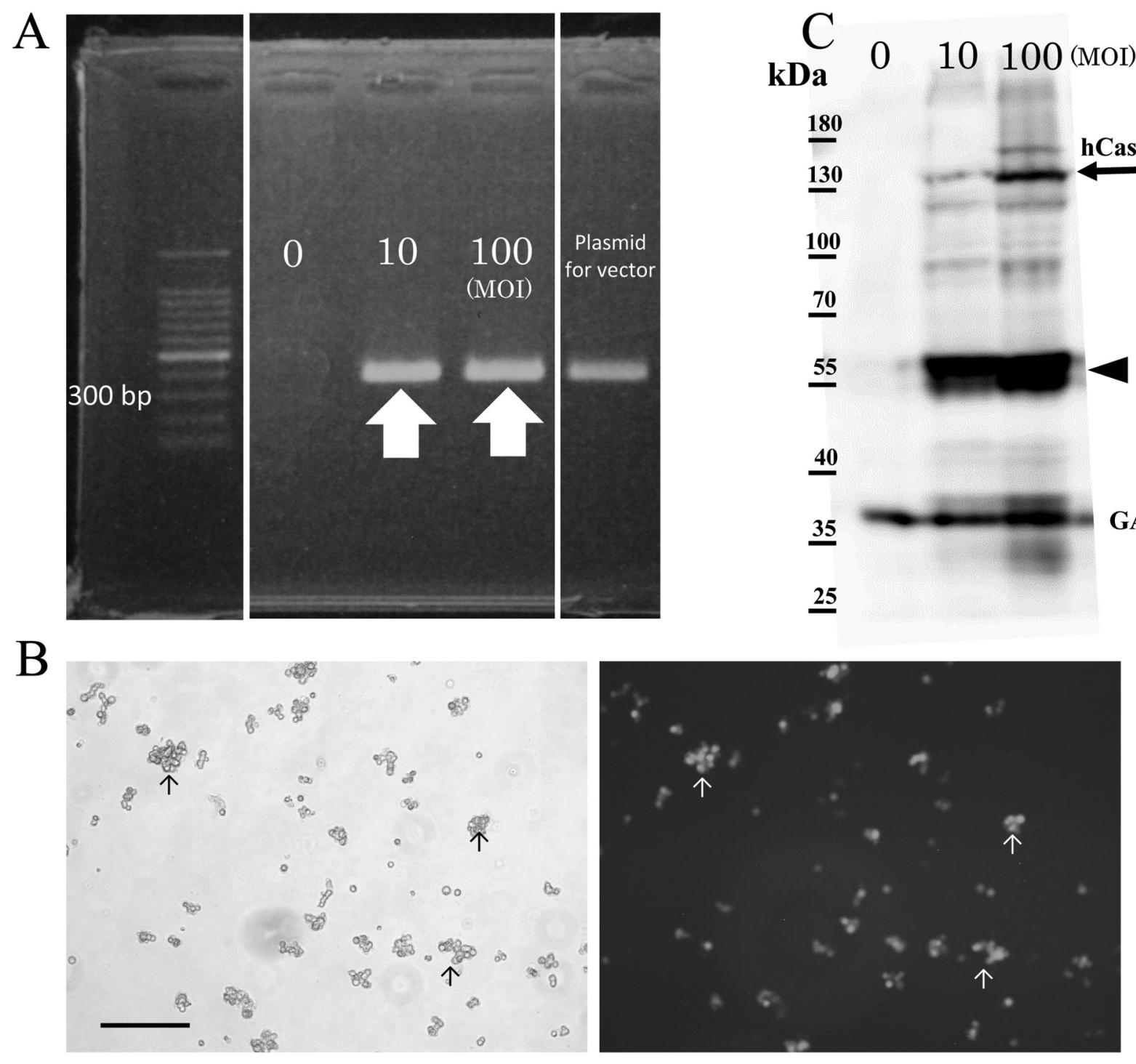

Figure 3. Infection of HEK293 bladder cancer cells by chimeric Ad5F35 vector containing the Cas 9 gene regulated by the midkine promoter (Ad5F35-Mkp-hCas9). A: Polymerase chain reaction (PCR) results for HEK293 cells $72 \mathrm{~h}$ after infection. PCR products of about 300 bp were detected in cells infected with Ad5F35-Mkp-hCas9 at 10 and 100 multiplicity of infection (MOI) (arrows), and these PCR products were in the same position as the PCR products from the plasmid for the vector. No PCR products were detected in control HEK293 cells. B: Fluorescence of enhanced green fluorescent protein $72 \mathrm{~h}$ after infection of HEK293 cells with $100 \mathrm{MOI}$ of Ad5F35-Mkp-hCas 9. HEK293 cells appeared round, and many cells were floating but strong green fluorescence was observed (arrows; bar=250 $\mu \mathrm{m}$ ). The brightness and contrast of these photos have been adjusted. C: After 96 h from Ad5F35-Mkp-hCas9 infection, two specific signals of Ad5F35-Mkp-hCas9-infected HEK293 cells near 130-150 kDa (arrow), and near $55 \mathrm{kDa}$ (arrowhead) were detected by western blotting. The band of higher molecular weight was considered to be the hCas 9 protein because the molecular weight of hCas9 was calculated to be 164,649 Da. GAPDH: Glyceraldehyde 3-phosphate dehydrogenase.

1,000 MOI of Ad5F35-MKp-hCas9. At 100 MOI, the survival rate of PNT1A cells was lower than that of the control but not significantly different by the KruskalWallis rank-sum test. On the other hand, significantly higher viability than that of control was observed at 10 MOI (Figure 2A).
In UMUC3 cells, significant survival inhibition was observed at 100 and 1,000 MOI of Ad5F35-MKp-hCas9. The survival rate at $10 \mathrm{MOI}$ was lower than that of the control but no significant difference was detected by two-way analysis of variance (Figure 2B). In 253J cells, 100 and 1,000 MOI of Ad5F35-MKp-hCas9 significantly reduced the 


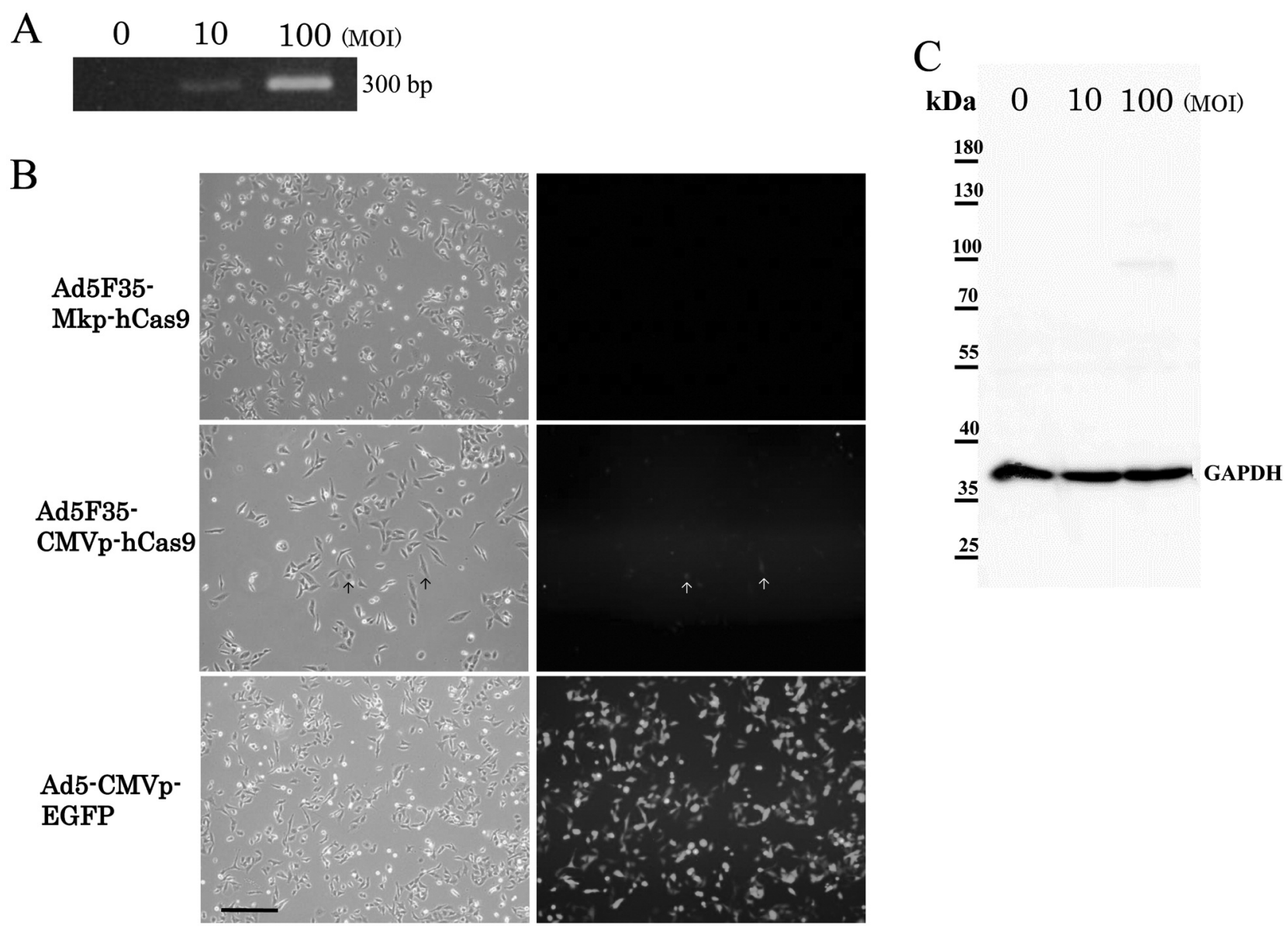

Figure 4. Vector-infected non-malignant PNT1A cells. A: Polymerase chain reaction product of human Cas9 (hCas9) DNA was confirmed 72 h after infection with Ad5F35 vector containing the Cas 9 gene regulated by the midkine promoter (Ad5F35-Mkp-hCas9) at both 10 and 100 multiplicity of infection (MOI). B: Intracellular fluorescence of enhanced green fluorescent protein in PNT1A cells at $72 \mathrm{~h}$ after infection at $100 \mathrm{MOI}$ with Ad5F35Mkp-hCas9, Ad5F35 vector containing the Cas 9 gene under regulation by cytomegalovirus promoter (CMVp) (Ad5F35-CMVp-hCas9), and Ad5CMVp-enhanced green fluorescent protein (EGFP). No fluorescence of EGFP was observed in Ad5F35-Mkp-hCas9-infected cells. Very faint fluorescence was observed in Ad5F35-CMVp-hCas9-infected cells (arrows). Many PNT1A cells that emitted strong fluorescence were observed after infection with Ad5-CMVp-EGFP. Bar=250 $\mu \mathrm{m}$. The brightness and contrast of these photos have been adjusted. C: No hCas 9 signals were detected by western blotting from Ad5F35-Mkp-hCas9-infected PNT1A cells. GAPDH: Glyceraldehyde 3-phosphate dehydrogenase.

survival rate but no significant difference was observed at 10 MOI of Ad5F35-Mkp-hCas9 (Figure 2C). Similarly to UMUC3 and 253J cells, 100 and 1,000 MOI of Ad5F35Mkp-hCas 9 significant reduced the survival rate of 5637 cells. On the other hand, the survival rate at $10 \mathrm{MOI}$ of Ad5F35-Mkp-hCas9 was not significantly different from that of the control cells (Figure 2D).

hCas9 expression in each cell line. PCR products with a length of approximately 300 bp were detected in Ad5F35Mkp-hCas9 infected HEK293 cells at both 10 and 100 MOI. These PCR products were detected at the same position as the PCR products obtained from the plasmid vector. In contrast, no PCR products were detected in the control HEK 293 cells (Figure 3A). Intracellular EGFP expression was observed from $24 \mathrm{~h}$ after infection with Ad5F35-Mkp-hCas9 at both 10 and 100 MOI. At $72 \mathrm{~h}$ after infection, all HEK293 cells appeared round and many cells were floating but strong EGFP fluorescence was observed (Figure 3B). Western blots revealed two bands that were specific to Ad5F35-MkphCas9-infected HEK293 cells (Figure 3C). One band was between 130-150 kDa (arrow), and the other was near 55 $\mathrm{kDa}$ (arrowhead). The hCas9 protein used in this study was 1,423 amino acids long, and the calculated molecular weight was 164,649 Da. Therefore, the band of higher molecular weight was considered to be the hCas 9 protein. 

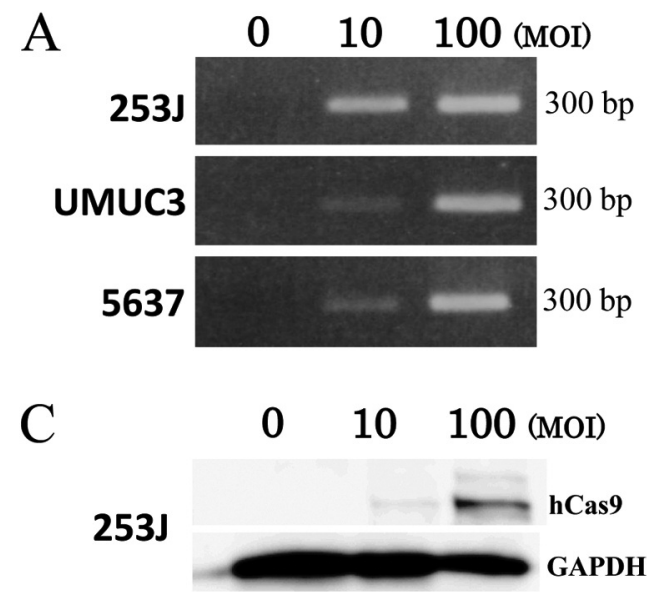

UMUC3

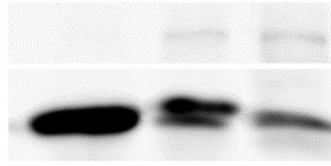

hCas9

GAPDH

5637

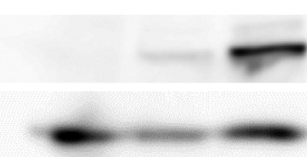

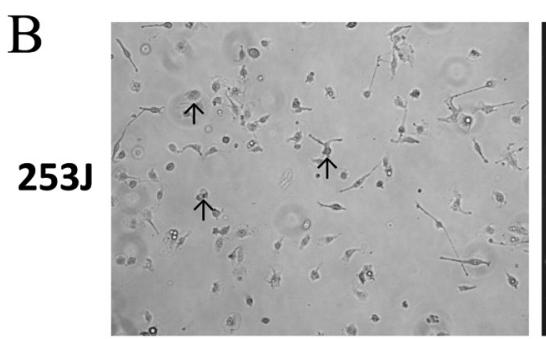

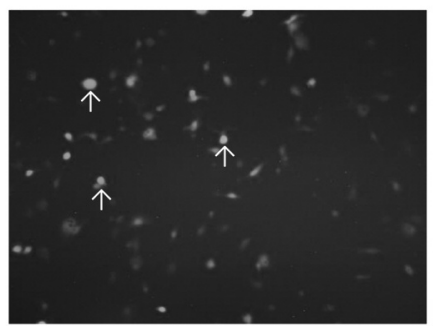

UMUC3
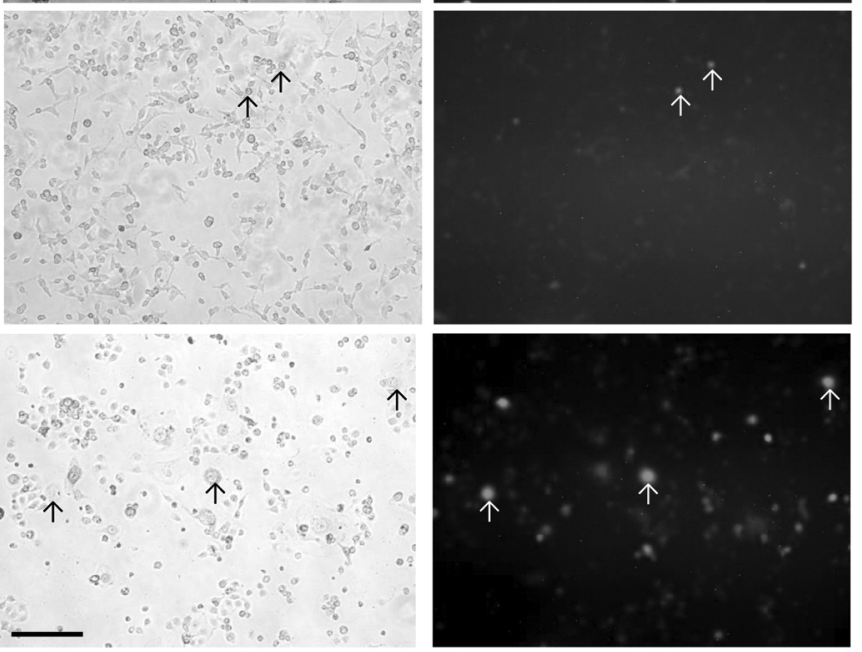

Figure 5. Infection of bladder cancer cells with Ad5F35 vector containing the Cas 9 gene regulated by the midkine promoter (Ad5F35-Mkp- $h$ Cas 9$)$. A: Polymerase chain reaction (PCR) analysis of hCas 9 expression at 72 h from infection. PCR products of hCas 9 DNA were detected in all experimental ceIl lines. B: Fluorescence enhanced green fluorescent protein (EGFP) 72 h after infection at 100 multiplicity of infection (MOI) of Ad5F35-Mkp-hCas9. $253 \mathrm{~J}$ and 5637 cells showed round shape and strong EGFP fluorescence was observed (arrows; bar=250 $\mu \mathrm{m}$ ). On the other hand, UMUC3 cells maintained a radial shape and EGFP fluorescence was dim (arrows). The brightness and contrast of these photos have been adjusted. C: hCas 9 protein expression in the bladder cancer cells at 96 h from infection. GAPDH: Glyceraldehyde 3-phosphate dehydrogenase.

In PNT1A cells that were infected with Ad5F35-MkphCas9, a 300-bp PCR product was also observed. The PCR signal of $100 \mathrm{MOI}$-infected PNT1A cells was stronger than that of 10 MOI-infected cells (Figure 4A). However, no EGFP fluorescence was observed in infected PNT1A cells, even at $100 \mathrm{MOI}$ of Ad5F35-Mkp-hCas9 (Figure 4B). On the other hand, Ad5F35-CMVp-hCas9-infected PNT1A cells showed a glimmer of fluorescence, and Ad5-CMVp-EGFPinfected PNT1A showed strong EGFP fluorescence (Figure 4B). No significant signals of hCas9 were detected by western blotting in Ad5F35-Mkp-hCas9-infected-PNT1A (Figure 4C).

hCas 9 expression in bladder cancer cell lines. PCR products were detected in all experimental bladder cancer cells (253J, UMUC3, and 5637) after Ad5F35-Mkp-hCas9 infection (Figure 5A). In general, the PCR signals of the 100 MOIinfected cells were stronger than those of the 10 MOIinfected cells.
Intracellular EGFP expression in the bladder cancer cells after $72 \mathrm{~h}$ of Ad5F35-Mkp-hCas9 infection at $100 \mathrm{MOI}$ is shown in Figure 5B. At 100 MOI, EGFP fluorescence was observed from $24 \mathrm{~h}$ after infection in all cell lines but no EGFP expression was observed at 10 MOI. In 253J and 5637 cells, EGFP fluorescence was strong, and many cells appeared round in shape. In contrast, UMUC3 cells showed faint EGFP fluorescence and maintained a radial shape even $72 \mathrm{~h}$ after infection with Ad5F35-Mkp-hCas9.

Western blotting revealed hCas9-specific signals in Ad5F35-Mkp-hCas9-infected bladder cancer cells (Figure 5C) at both 10 and $100 \mathrm{MOI}$. The average expression level of hCas9 protein at $72 \mathrm{~h}$ after Ad5F35-Mkp-hCas9 infection is shown in Figure 6. No hCas9 expression was observed in PNT1A cells. In the bladder cancer cell lines, the hCas9 expression level was highest in HEK293 cells. hCas9 expression of 100 MOI-infected cells was higher than that of 10 MOI-infected cells except HEK293, although without significance. The hCas9 protein level was 


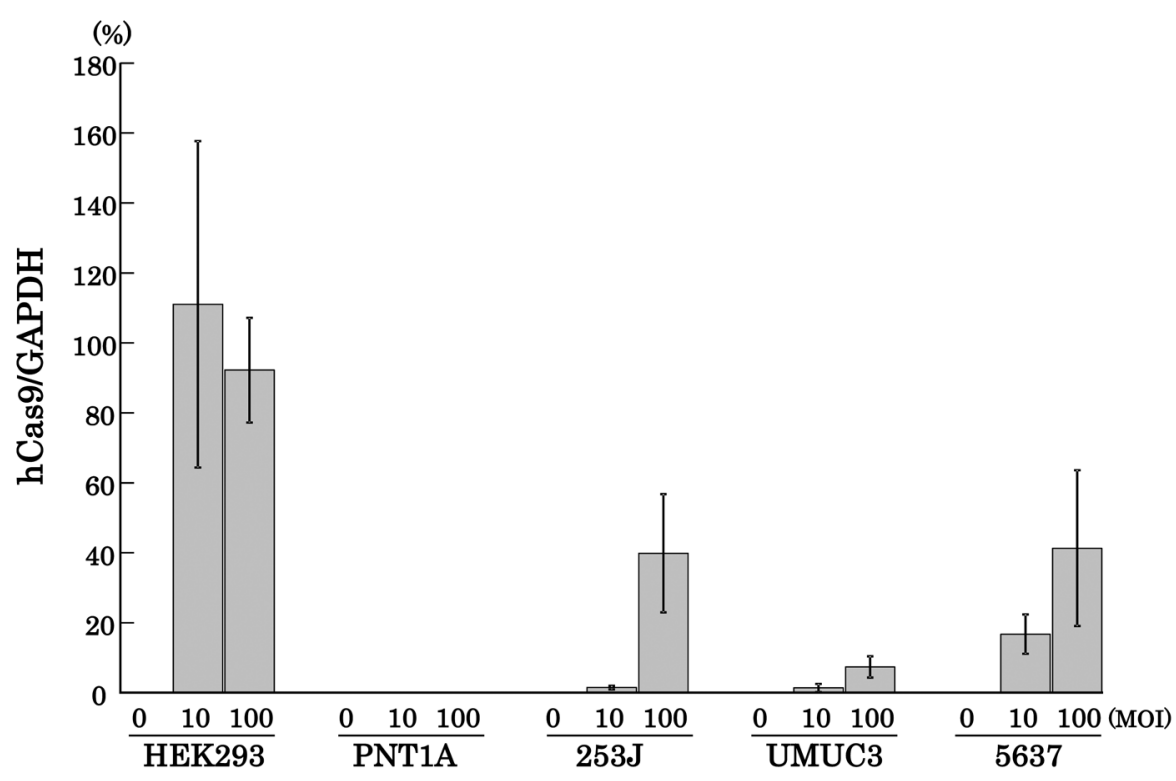

Figure 6. Relative expression levels of hCas 9 protein in the experimental cell lines after infection with Ad5F35 vector containing the Cas 9 gene regulated by the midkine promoter (Ad5F35-Mkp-hCas9). PNT1A cells showed no hCas 9 expression. After infection with Ad5F35-Mkp-hCas 9 at 10 multiplicity of infection (MOI), 5637 cells showed relatively higher hCas9 expression than 253J and UNUC3 cells. At 100 MOI, $253 \mathrm{~J}$ and 5637 cells showed higher hCas9 expression than UMUC3 cells. GAPDH: Glyceraldehyde 3-phosphate dehydrogenase.

moderate in $253 \mathrm{~J}$ and 5637 cells but relatively low in UMUC3 cells.

\section{Discussion}

This study examined whether it was possible to achieve cancer cell-specific hCas9 gene expression using the Ad5F35 vector and midkine promoter. The Ad5F35 vector is a chimeric adenovirus vector in which the Ad5 fiber knob is replaced with the Ad35 fiber knob $(4,6,7)$. Ad5 infects cells bearing coxsackie and adenovirus receptors (30) but expression of such receptors is relatively low in malignant cells $(4,31)$. In contrast, Ad35 infects cells with CD46, which is more highly expressed in malignant cells (31). Midkine is a heparin-binding growth factor that is involved in various functions such as mitogenesis, angiogenesis, anti-apoptosis, fibrinolysis, and transformation (32). Increased midkine expression has been observed in many types of cancer cells (8). Therefore, the midkine promoter should be applied to express genes that are useful for cancer treatment specifically in tumor cells $(4,33)$.

The data on cell viability and PCR suggest that the Ad5F35-Mkp-hCas9 vector infected the PNT1A cells. However, the expression of the hCas9 gene was not confirmed by EGFP fluorescence and western blotting results. In other words, in PNT1A, which is a non-malignant cell line, the gene was not expressed even in cells infected with Ad5F35-MkphCas9. On the other hand, faint fluorescence was observed in
Ad5F35-CMVp-hCas9-infected PNT1A cells, and strong green fluorescence was observed in PNT1A cells infected with Ad5-CMVp-EGFP. These observations indicate that the Ad5F35 vector is less infectious to PNT1A cells than the Ad5 vector, and that CMVp is an effective promoter in PNT1A cells. Taken together, similarly to our previous findings, we can conclude that the combination of Ad5F 35 and the midkine promoter minimizes the possibility of unintended gene expression in non-malignant cells.

In the bladder cancer cells used in this study, the expression of hCas9 protein was confirmed by EGFP fluorescence and western blotting after Ad5F35-Mkp-hCas9 infection. We already confirmed the expression of CD46 and midkine in the bladder cancer cell lines used for this experiment (4), and our present results are quite adequate. In the present study, the relative hCas9 expression levels at 253J and 5637 cells were higher than those of UMUC 3 cells, and the apparent intensity of EGFP fluorescence in each cell line also showed a similar tendency to the pattern of hCas9 expression. In our previous study, we investigated the mRNA expression of CD46 and midkine in various bladder cancer cell lines and found that UMUC3 cells showed the lowest mRNA expression levels of both. Therefore, the expression level of hCas9 is considered to correlate well with the expression levels of CD46 and midkine mRNAs.

Cell viability data showed that 100 MOI of Ad5F35-MkphCas9 was toxic to non-malignant cells. In contrast, hCas9 
expression in bladder cancer cells was measurable even at $10 \mathrm{MOI}$, thus suggesting that it should be used at lower than 100 MOI for clinical application.

Although the CRISPR-Cas9 system is considered a promising strategy for novel gene therapy, there are serious concerns that unintended gene mutations due to off-target effects may exacerbate cancer. Some studies have suggested that the frequency of off-target mutations with the CRISPRCas9 system will reach $40-80 \%(22,34)$. Currently, various studies are in progress to minimize the risk of these offtarget mutations (25-27), and several methods using tissuespecific promoters have already been attempted to express Cas9 endonuclease only in target cells (27). Our results showed that the midkine promoter can specifically express the Cas9 gene in bladder cancer cells.

The Ad5F35-Mkp-hCas9 vector did not carry a guide RNA sequence. This is considered to reduce the risk of unwanted gene editing compared to a vector containing Cas9 and a guide RNA. In addition, the range of application can be easily expanded simply by changing only the guide RNA-vector, and we consider that this might provide a new strategy for the treatment of refractory bladder cancer. For example, anti-angiogenic therapy generally lacks selectivity for tumor tissues. However, cancer cell-specific anti-angiogenic therapy may be achieved by the combination of the Ad5F35-Mkp-hCas9 vector and guide RNA targeting vascular endothelial growth factor A.

\section{Conclusion}

The results of the present study showed that the Ad5F35MKp-hCas9 vector is capable of expressing the hCas9 gene with high specificity in bladder cancer cells. We believe these findings may help in minimizing the risk of off-target effects of gene editing.

\section{Conflicts of Interest}

The Authors have no conflicts of interest to report in relation to this study.

\section{Authors' Contributions}

Conception and design: W.M. and A.G. Development of methodology: W.M., K.H., M.T. and T.M. Acquisition of data: W.M. Analysis and interpretation of data: W.M. Writing, review, and/or revision of the article: W.M. and A.G. Administrative, technical, or material support: W.M. and A.G. Study supervision: A.G.

\section{Acknowledgements}

This work was supported by JSPS KAKENHI Grant Number 19K09681.

\section{References}

1 National Cancer Center web site. Available at: https:// ganjoho.jp/reg_stat/statistics/stat/summary.html [Last accessed on June 30th, 2021]

2 Leopardo D, Cecere SC, Di Napoli M, Cavaliere C, Pisano C, Striano S, Marra L, Menna L, Claudio L, Perdonà S, Setola S, Berretta M, Franco R, Tambaro R, Pignata S and Facchini G: Intravesical chemo-immunotherapy in non muscle invasive bladder cancer. Eur Rev Med Pharmacol Sci 17(16): 2145-2158, 2013. PMID: 23893180.

3 Takao Y, Masumoto N, Takasu Y, Ohtsuki H, Wadagaki F, Kajio $\mathrm{K}$, Yoshimoto $\mathrm{T}$ and Iwakawa $\mathrm{S}$ : Comparison of subjective adverse effects, accomplishment rate and achievement rate during intravesical instillation therapy using BCG (Bacillus Calmette-Guerin) or pirarubicin for bladder cancer patients at Kawanishi City General Hospital. Iryo Yakugaku (Japanese Journal of Pharmaceutical Health Care and Sciences) 32(3): 235241, 2021. DOI: 10.5649/jjphcs.32.235

4 Gotoh A, Nagaya H, Kanno T, Tagawa M and Nishizaki T: Fiber-substituted conditionally replicating adenovirus Ad5F35 induces oncolysis of human bladder cancer cells in in vitro analysis. Urology 81(4): 920.e7-920.11, 2013. PMID: 23394881. DOI: $10.1016 /$ j.urology.2012.12.023

5 Matsunaga W, Ichikawa M, Nakamura A, Ishikawa T and Gotoh A: Lentiviral vector-mediated gene transfer in human bladder cancer cell lines. Anticancer Res 38(4): 2015-2020, 2018. PMID: 29599318. DOI: 10.21873 /anticanres. 12440

6 Acharya B, Terao S, Suzuki T, Naoe M, Hamada K, Mizuguchi $\mathrm{H}$ and Gotoh $\mathrm{A}$ : Improving gene transfer in human renal carcinoma cells: Utilization of adenovirus vectors containing chimeric type 5 and type 35 fiber proteins. Exp Ther Med 1(3): 537-540, 2010. PMID: 22993573. DOI: 10.3892/etm_ 00000085

7 Kanno T, Gotoh A, Nakano T, Tagawa M and Nishizaki T: Beneficial oncolytic effect of fiber-substituted conditionally replicating adenovirus on human lung cancer. Anticancer Res 32(11): 4891-4895, 2012. PMID: 23155257.

8 Kato M, Maeta $\mathrm{H}$, Kato $\mathrm{S}$, Shinozawa $\mathrm{T}$ and Terada $\mathrm{T}$ : Immunohistochemical and in situ hybridization analyses of midkine expression in thyroid papillary carcinoma. Mod Pathol 13(10): 1060-1065, 2000. PMID: 11048798. DOI: 10.1038/modpathol. 3880195

9 Nwanegbo E, Vardas E, Gao W, Whittle H, Sun H, Rowe D, Robbins PD and Gambotto A: Prevalence of neutralizing antibodies to adenoviral serotypes 5 and 35 in the adult populations of The Gambia, South Africa, and the United States. Clin Diagn Lab Immunol 11(2): 351-357, 2004. PMID: 15013987. DOI: $10.1128 /$ cdli.11.2.351-357.2004

10 Chen Y, Yu DC, Charlton D and Henderson DR: Pre-existent adenovirus antibody inhibits systemic toxicity and antitumor activity of CN706 in the nude mouse LNCaP xenograft model: implications and proposals for human therapy. Hum Gene Ther 11(11): 1553-1567, 2000. PMID: 10945769. DOI: 10.1089/1043 0340050083289

11 Varela JC, Atkinson C, Woolson R, Keane TE and Tomlinson S: Upregulated expression of complement inhibitory proteins on bladder cancer cells and anti-MUC1 antibody immune selection. Int J Cancer 123(6): 1357-1363, 2008. PMID: 18561323. DOI: $10.1002 / \mathrm{ijc} .23676$ 
12 Small JC, Haut LH, Bian A and Ertl HC: The effect of adenovirus-specific antibodies on adenoviral vector-induced, transgene product-specific T cell responses. J Leukoc Biol 96(5): 821-831, 2014. PMID: 25082150. DOI: 10.1189/jlb.1A0813451RR

13 Heo J, Reid T, Ruo L, Breitbach CJ, Rose S, Bloomston M, Cho M, Lim HY, Chung HC, Kim CW, Burke J, Lencioni R, Hickman T, Moon A, Lee YS, Kim MK, Daneshmand M, Dubois K, Longpre L, Ngo M, Rooney C, Bell JC, Rhee BG, Patt R, Hwang TH and Kirn DH: Randomized dose-finding clinical trial of oncolytic immunotherapeutic vaccinia JX-594 in liver cancer. Nat Med 19(3): 329-336, 2013. PMID: 23396206. DOI: $10.1038 / \mathrm{nm} .3089$

14 Pilankatta R, Chawla T, Khanna N and Swaminathan S: The prevalence of antibodies to adenovirus serotype 5 in an adult Indian population and implications for adenovirus vector vaccines. J Med Virol 82(3): 407-414, 2010. PMID: 20087930. DOI: $10.1002 / j m v .21721$

15 Logunov DY, Dolzhikova IV, Zubkova OV, Tukhvatulin AI, Shcheblyakov DV, Dzharullaeva AS, Grousova DM, Erokhova AS, Kovyrshina AV, Botikov AG, Izhaeva FM, Popova O, Ozharovskaya TA, Esmagambetov IB, Favorskaya IA, Zrelkin DI, Voronina DV, Shcherbinin DN, Semikhin AS, Simakova YV, Tokarskaya EA, Lubenets NL, Egorova DA, Shmarov MM, Nikitenko NA, Morozova LF, Smolyarchuk EA, Kryukov EV, Babira VF, Borisevich SV, Naroditsky BS and Gintsburg AL: Safety and immunogenicity of an rAd26 and rAd5 vector-based heterologous prime-boost COVID-19 vaccine in two formulations: two open, non-randomised phase $1 / 2$ studies from Russia. Lancet 396(10255): 887-897, 2020. PMID: 32896291. DOI: 10.1016/S0140-6736(20)31866-3

16 Deltcheva E, Chylinski K, Sharma CM, Gonzales K, Chao Y, Pirzada ZA, Eckert MR, Vogel J and Charpentier E: CRISPR RNA maturation by trans-encoded small RNA and host factor RNase III. Nature 471(7340): 602-607, 2011. PMID: 21455174. DOI: $10.1038 /$ nature09886

17 Jinek M, Chylinski K, Fonfara I, Hauer M, Doudna JA and Charpentier E: A programmable dual-RNA-guided DNA endonuclease in adaptive bacterial immunity. Science 337(6096): 816-821, 2012. PMID: 22745249. DOI: 10.1126/science.1225829

18 Ran FA, Hsu PD, Wright J, Agarwala V, Scott DA and Zhang F: Genome engineering using the CRISPR-Cas9 system. Nat Protoc 8(11): 2281-2308, 2013. PMID: 24157548. DOI: 10.1038/nprot. 2013.143

19 Yao S, He Z and Chen C: CRISPR/Cas9-mediated genome editing of epigenetic factors for cancer therapy. Hum Gene Ther 26(7): 463-471, 2015. PMID: 26075804. DOI: 10.1089/hum.2015.067

20 Akram F, Ikram Ul Haq, Ahmed Z, Khan H and Ali MS: CRISPR-Cas9, a promising therapeutic tool for cancer therapy: a review. Protein Pept Lett 27(10): 931-944, 2020. PMID: 32264803. DOI: $10.2174 / 0929866527666200407112432$

21 Baylis F and McLeod M: First-in-human Phase 1 CRISPR gene editing cancer trials: Are we ready? Curr Gene Ther 17(4): 309-319, 2017. PMID: 29173170. DOI: $10.2174 / 1566523217666171121$ 165935

22 Fu Y, Foden JA, Khayter C, Maeder ML, Reyon D, Joung JK and Sander JD: High-frequency off-target mutagenesis induced by CRISPR-Cas nucleases in human cells. Nat Biotechnol 31(9): 822-826, 2013. PMID: 23792628. DOI: 10.1038/nbt.2623
23 Cho SW, Kim S, Kim Y, Kweon J, Kim HS, Bae S and Kim JS: Analysis of off-target effects of CRISPR/Cas-derived RNAguided endonucleases and nickases. Genome Res 24(1): 132141, 2014. PMID: 24253446. DOI: 10.1101/gr.162339.113

24 Corrigan-Curay J, O'Reilly M, Kohn DB, Cannon PM, Bao G, Bushman FD, Carroll D, Cathomen T, Joung JK, Roth D, Sadelain M, Scharenberg AM, von Kalle C, Zhang F, Jambou R, Rosenthal E, Hassani M, Singh A and Porteus MH: Genome editing technologies: defining a path to clinic. Mol Ther 23(5): 796-806, 2015. PMID: 25943494. DOI: 10.1038/mt.2015.54

25 Standage-Beier K, Zhang Q and Wang X: Targeted large-scale deletion of bacterial genomes using CRISPR-nickases. ACS Synth Biol 4(11): 1217-1225, 2015. PMID: 26451892. DOI: 10.1021/acssynbio.5b00132

26 Tsai SQ, Nguyen NT, Malagon-Lopez J, Topkar VV, Aryee MJ and Joung JK: CIRCLE-seq: a highly sensitive in vitro screen for genome-wide CRISPR-Cas9 nuclease off-targets. Nat Methods 14(6): 607-614, 2017. PMID: 28459458. DOI: 10.1038/ nmeth. 4278

27 Han HA, Pang JKS and Soh BS: Mitigating off-target effects in CRISPR/Cas9-mediated in vivo gene editing. J Mol Med (Berl) 98(5): 615-632, 2020. PMID: 32198625. DOI: 10.1007/s00109020-01893-Z

28 Uehara K, Matsubara S, Kadomatsu K, Tsutsui J and Muramatsu T: Genomic structure of human midkine (MK), a retinoic acidresponsive growth/differentiation factor. J Biochem 111(5): 563-567, 1992. PMID: 1639750. DOI: 10.1093/oxfordjournals.jbchem. a123797

29 Nagaya H, Gotoh A, Kanno T and Nishizaki T: A3 adenosine receptor mediates apoptosis in in vitro RCC4-VHL human renal cancer cells by up-regulating AMID expression. J Urol 189(1): 321328, 2013. PMID: 23174235. DOI: 10.1016/j.juro.2012.08.193

30 Hamilton MM, Byrnes GA, Gall JG, Brough DE, King CR and Wei LL: Alternate serotype adenovector provides long-term therapeutic gene expression in the eye. Mol Vis 14: 2535-2546, 2008. PMID: 19122827.

31 Gaggar A, Shayakhmetov DM and Lieber A: CD46 is a cellular receptor for group B adenoviruses. Nat Med 9(11): 1408-1412, 2003. PMID: 14566335. DOI: 10.1038/nm952

32 Sakamoto K and Kadomatsu K: Midkine in the pathology of cancer, neural disease, and inflammation. Pathol Int 62(7): 445-455, 2012. PMID: 22726064. DOI: 10.1111/j.1440-1827.2012.02815.x

33 Nagaya H, Tagawa M, Hiwasa K, Terao S, Kanno T, Nishizaki $\mathrm{T}$ and Gotoh A: Fiber-substituted conditionally replicating adenovirus for oncolysis of human renal carcinoma cells. Anticancer Res 32(7): 2985-2989, 2012. PMID: 22753762.

34 Carey K, Ryu J, Uh K, Lengi AJ, Clark-Deener S, Corl BA and Lee K: Frequency of off-targeting in genome edited pigs produced via direct injection of the CRISPR/Cas9 system into developing embryos. BMC Biotechnol 19(1): 25, 2019. PMID: 31060546. DOI: 10.1186/s12896-019-0517-7 